\title{
Power Independent EMG based Gesture Recognition for Robotics
}

\author{
Ling Li, David Looney, Cheolsoo Park, Naveed U. Rehman, and Danilo P. Mandic
}

\begin{abstract}
A novel method for detecting muscle contraction is presented. This method is further developed for identifying four different gestures to facilitate a hand gesture controlled robot system. It is achieved based on surface Electromyograph (EMG) measurements of groups of arm muscles. The crossinformation is preserved through a simultaneous processing of EMG channels using a recent multivariate extension of Empirical Mode Decomposition (EMD). Next, phase synchrony measures are employed to make the system robust to different power levels due to electrode placements and impedances. The multiple pairwise muscle synchronies are used as features of a discrete gesture space comprising four gestures (flexion, extension, pronation, supination). Simulations on real-time robot control illustrate the enhanced accuracy and robustness of the proposed methodology.
\end{abstract}

\section{INTRODUCTION}

The study of Electromyograph (EMG) signals has been an active area for analyzing the biomechanics of human muscle for various clinical and biomedical applications; these include the clinical diagnosis of abnormal muscle movement and behavior in generating control signals for electronic equipment (e.g. electric wheelchair, mobile robot, game devices), as a human computer interface. However, in such applications, one often comes across the multichannel EMG signals whose dynamics and the associated couplings between subsets of muscles is difficult to model, especially when performing tasks like robot steering etc. Existing methods have modelled EMG activity based on the difference in power levels measured at select muscle locations [1]. However, such approaches are critically sensitive to electrode placement, making it difficult to develop a general analysis framework which is suitable for several subjects. Furthermore, changing electrode impedance caused by human motion, power line interference, and the conductivity of the electrode gel makes power based features unreliable in practice. Another challenge for the energy based method includes muscle fatigue; the design of appropriate detection, estimation algorithms, and robust feature extraction methods remains a key prerequisite to accurate state classification.

The effectiveness of phase synchrony features for modelling couplings between different regions of the brain has been established in the literature [2]. The feature is power independent, robust to electrode placement, and can, therefore, be used to deal with muscle fatigue and false detection of muscle contraction. A framework for estimating phase synchrony in EEG using EMD [3] was proposed in [4]. The

Manuscript received March 23, 2011.

L. Li, D. Looney, N. Rehman and D. P. Mandic are with the Department of Electrical and Electronic Engineering, Imperial College London, London SW7 2AZ, U.K. (e-mail: ling.li206@imperial.ac.uk).
EMD algorithm adaptively decomposes a given signal into a number of oscillatory components which are, by design, narrow band making it possible to obtain highly localised phase information using the Hilbert transform. As the approach makes no prior assumptions of the signal, it provides a robust synchrony estimation framework [5] for nonlinear and nonstationary data. Recently, it has been shown that the complex extensions [6], [7] of the algorithm facilitate higher accuracies in determining phase and frequency information in the presence of noise.

In this paper, a novel method for detecting muscle contraction is proposed to perform robust gesture recognition (extension, flexion, pronation, and supination of the arm) for EMG data using phase synchrony features. Especially, the phase synchrony analysis using the recent multivariate extensions of EMD (MEMD) [8] [9] has been presented. Both bivarite EMD (BEMD) [7] and MEMD are used for phase analysis to identify muscle contraction. It is shown how the proposed methodology can simultaneously model couplings between electrodes facilitating muscle activity detection and enhanced gesture recognition.

\section{METHODOLOGY}

\section{A. EMD Algorithm and its Multivariate Extensions}

EMD is a data driven technique that can be used to decompose a signal into a number of zero-mean, band limited oscillatory components, called intrinsic mode functions (IMFs) [3]. Due to the data driven nature of EMD, it has been frequently employed for processing real world non-stationary signals. Recently, several multivariate extensions of EMD have been developed, of which multivariate extension of EMD (MEMD) [8] is particularly useful, as it can decompose a multivariate signal containing any number of channels. It is based on the concept of calculating the local mean of the input signal via multiple signal projections; since the input signal resides in $\mathrm{n}$-dimensional space, projections of the input signal are, therefore, taken along the uniform pointset in $\mathrm{n}$ dimensional space based on the low discrepancy Hammersley sequence [8].

Consider a sequence of $\mathrm{N}$-dimensional vectors $\{\mathbf{v}(t)\}_{t=1}^{T}=\left\{v_{1}(t), v_{2}(t), \ldots, v_{N}(t)\right\}$ representing a multivariate signal with $N$ components, and $\mathrm{x}^{\theta_{k}}=$ $\left\{x_{1}^{k}, x_{2}^{k}, \ldots, x_{N}^{k}\right\}$ denoting a set of direction vectors along the directions given by angles $\theta^{k}=\left\{\theta_{1}^{k}, \theta_{2}^{k}, \ldots, \theta_{(N-1)}^{k}\right\}$ on an (n-1)-sphere. Then the extraction of first IMF from the proposed multivariate extension of EMD is summarized as:

1) Choose a pointset for sampling on an (n-1)-sphere;

2) Calculate a projection, denoted by $\left\{p^{\left(\theta_{k}\right)}(t)\right\}_{t=1}^{T}$, of the input signal $\{\mathbf{v}(t)\}_{t=1}^{T}$ along the direction vector 
$\mathbf{x}^{\left(\theta_{k}\right)}$, for all $k$ (the whole set of direction vectors), giving $\left\{p^{\left(\theta_{k}\right)}(t)\right\}_{k=1}^{K}$ as the set of projections;

3) Find the time instants $\left\{t_{i}^{\left(\theta_{k}\right)}\right\}$ corresponding to the maxima of the set of projected signals $\left\{p^{\left(\theta_{k}\right)}(t)\right\}_{k=1}^{K}$;

4) Interpolate $\left[t_{i}^{\left(\theta_{k}\right)}, \mathbf{v}\left(t_{i}^{\left(\theta_{k}\right)}\right)\right]$ to obtain multivariate envelope curves $\left\{\mathbf{e}^{\left(\theta_{k}\right)}(t)\right\}_{k=1}^{K}$;

5) For a set of $K$ direction vectors, the mean $\mathbf{m}(t)$ of the envelope curves is calculated as: $\mathbf{m}(t)=$ $\frac{1}{K} \sum_{k=1}^{K} \mathbf{e}^{\left(\theta_{k}\right)}(t)$

6) Extract the "detail" $d(t)$ using $d(t)=x(t)-m(t)$. If the "detail" fulfills the stopping criterion for a multivariate IMF, apply the above procedure to $x(t)-d(t)$, otherwise apply it to $d(t)$.

Hilbert transform [10] can then be applied to each IMF to extract the instantaneous frequency and amplitude; an analytic signal can be obtained by $z_{a}(t)=x(t)+j \tilde{x}(t)$, where $\tilde{x}(t)$ represent the Hilbert transform of $x(t)$. The magnitude function $a(t)$ and phase function $\theta(t)$ are given by: $a(t)=\sqrt{x^{2}(t)+\tilde{x}^{2}(t)}$ and $\theta(t)=\arctan \left(\frac{\tilde{x}(t)}{x(t)}\right)$.

\section{B. The Phase Synchrony Index}

Phase synchrony models shared dynamics between that IMFs obtained for two sources. A straightforward way to obtain the IMFs is to apply real-valued EMD to each of the sources separately. However there are advantages [8] in decomposing sources simultaneously using complex or multivariate extensions of the algorithm, as they emphasize shared dynamics among the sources. For example, if there are two sources available, $x_{1}$ and $x_{2}$, BEMD can be applied to $z=x_{1}+j x_{2}$ to obtain a single set of complex IMFs. For two or more than two sources, MEMD can be used to decompose multiple sources simultaneously into multivariate IMFs. In this way, any shared signal dynamics that exists between the electrodes are better emphasized at the IMF level.

For the IMFs obtained for two sources, either within a BEMD or MEMD framework, phase synchrony is estimated as follows. The instantaneous amplitudes for the two sets of IMFs at each time instant $t=1, \ldots, T$ are denoted by $a_{i}(t)$ and $b_{i}(t)$, where $i=1, \ldots, M$ denotes the IMF index. For the $i$ th IMF, the instantaneous phase difference between the IMFs is given by $\phi_{i}(t)$. The distribution of $\phi_{i}(t)$ indicates the degree of phase synchrony. Deviation from the $\delta$ distribution (perfect synchrony) can be quantified by the phase coherence value (PCV) [11]: $\rho_{i}(t)=\frac{E_{\max }-E}{E_{\max }}$, where $E=-\sum_{n=1}^{N} p_{n} \ln p_{n}$, the Shannon's entropy of the distribution function $\phi_{i}\left(t-\frac{W}{2}: t+\frac{W}{2}\right)$ defined by a window length $W$, the number of bins $N$, the probability of $\phi_{i}\left(t-\frac{W}{2}: t+\frac{W}{2}\right)$ within $n$th bin $p_{n}$ [11]. The maximum entropy is $E_{\max }=0.626+0.4 \ln (W-1)$. In the process of evaluating the phase difference distribution, the power of the inputs is also be considered to cater for component relevance.

$$
\rho_{i}(t)=\left\{\begin{array}{cl}
\frac{E_{\max }-E}{E_{\max }}, & \text { if } a_{i}(t) \geq \epsilon, b_{i}(t) \geq \epsilon \\
0, & \text { if } a_{i}(t)<\epsilon, b_{i}(t)<\epsilon
\end{array}\right.
$$

where $\epsilon$ is an appropriate threshold. In this way, the degree of the phase synchrony is quantified by $\rho_{i}(t)$, which varies between 0 and 1 .

\section{EXPERIMENTAL RESULTS}

The purpose of the experiment is to control the robot movement of stop, moving forward, turning left, and turning right using gestures of extension, flexion, pronation, and supination, the EMG signal was recorded from 10 subjects using a single arm and hand performing a series of gestures (relaxed $\rightarrow$ extension $\rightarrow$ relaxed $\rightarrow$ flexion $\rightarrow$ relaxed $\rightarrow$ pronation $\rightarrow$ relaxed $\rightarrow$ supination $\rightarrow$ relaxed). Four surface muscles were measured using eight channels, with every two channels measuring a muscle. The signals obtained from the electrodes was digitalized (sampling frequency $4800 \mathrm{~Hz}$ ) and fed into a laptop via either a USB cable or Bluetooth connection for signal storage and analysis. After the processing of the data using Matlab, a control signal is generated and sent to the mobile robot for robot steering. In MEMD based simulations, we have used $K=128$ direction vectors for taking signal projections and the stopping criteria used is given in [12].

Fig. 1(a) illustrates the raw data obtained for channel 1 (top) and channel 2 (bottom) of the recorded EMG signal of subject 1 in time. Low frequency baseline effect can be seen clearly in both channels, especially the drift from sample number 6000 to 8000 in channel 2. These baseline was removed before phase synchrony analysis. The Ch1 and $\mathrm{Ch} 2$ of data are both measuring the 'extensor digitorum (ED)' muscle activity. Medical reference [13] shows that this muscle only tenses while extending the wrist or medical four fingers. A robust feature will only detect muscle contraction within the time interval which corresponds to the gesture 'extension'.

The top panel of Fig. 1(b) shows the total PCV obtained using BEMD. The parameter for calculating PCV was $W$ $=100$, and $N=100$. For rigor, a straightforward threshold was applied to the averaged total PCV for every 48 samples to obtain with 1 indicating contraction and 0 indicating no activity, the results of which are shown in the lower panel of Fig. 1(b). The same parameters were used to calculate PCV using MEMD approach. Result shown in Fig. 1(c) clearly suggests an improved accuracy using MEMD when the muscle is not tensed (e.g. from sample number 1500 to 2500). Note the MEMD approach also has a better performance while dealing with the drift effect in the action of 'supination'.

The muscle contraction detection ability and enhanced accuracy of the MEMD approach is confirmed by analyzing the EMG data of other muscles. Fig. 2 shows the 'flexor carpi radialis (FCR)' muscle activity. As suggested in [13], the muscle contraction should only be detected in the gesture of 'flexion' (flexes the wrist) and 'pronation' (abducts the wrist). Comparing Fig. 2(b) with Fig. 2(c), it can be seen both BEMD and MEMD based phase synchrony analysis can detect the muscle contraction, whereas by using the MEMD 

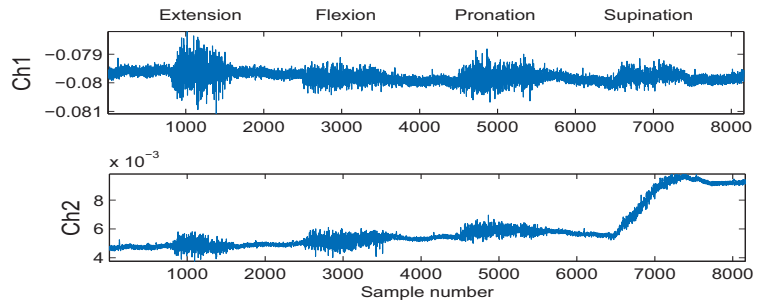

(a) Input signal - 'extensor digitorum' (channel 1 and 2)

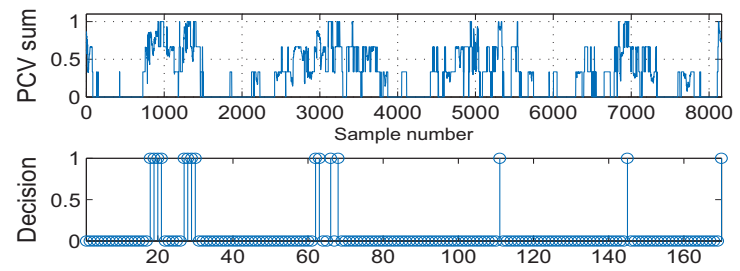

(b) BEMD phase synchrony - 'extensor digitorum' (channel 1 and 2)
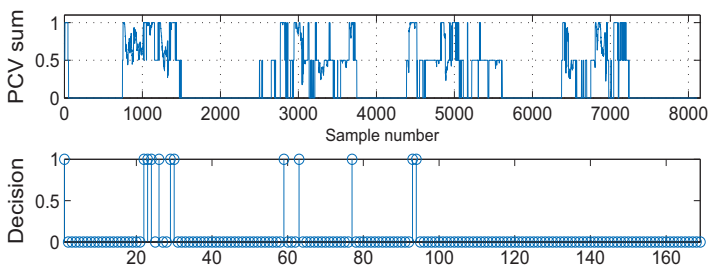

(c) MEMD phase synchrony - 'extensor digitorum' (channel 1 and 2)

Fig. 1. EMG data, phase synchrony, and the decision for muscle - 'extensor digitorum (ED)' between BEMD and MEMD method.

approach both the total PCV and decision result outperforms those using BEMD method.

In Fig. 3, the results of phase synchrony detection of the 'pronator teres (PR)' muscle are shown. The muscle contraction should be detected while performing 'extension' (flexes elbow) and 'pronation' (pronates forearm) [13]. There are indication of wrong decisions for both BEMD and MEMD approach. However, both BEMD and MEMD based method detect the muscle contraction. Same approach was used for the analysis of 'biceps brachii (BB)' muscle which is tensed in the action of both 'flexion' (flexor of forearm) and 'supination' (supination of forearm). Fig. 4(c) using MEMD approach detected the muscle tense for both gestures. On the contrary, the contraction of the muscle while performing 'flexion' was poorly recognized using the BEMD method as it is shown in Fig. 4(b).

Next, Table I was employed for the gesture recognition, with ' 1 ' indicating muscle contraction. States 1 to 5 in the table corresponding to the detection of gesture of extension (State 1: 1010), flexion (State 2: 0101), pronation (State 3: 0110), supination (State 4: 0001), and no specific gestures (State 5: all other combinations). The action index $(1,2,3$, $4,0)$ of states 1 to 5 were sent to the robot for the control of robot movement of stop, straight, left, right, and no action. The action index used for robot steering is shown in Fig. 5.

According to the medical reference [13], ED and PT
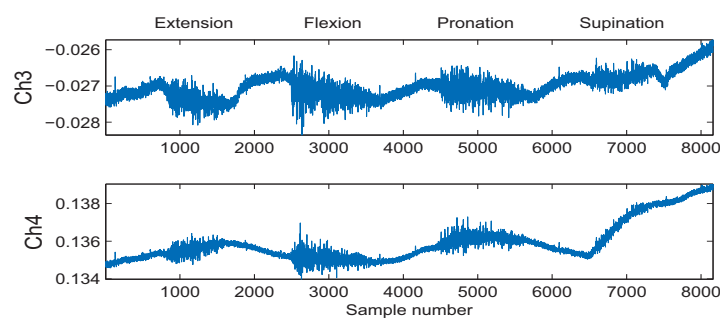

(a) Input signal - 'flexor carpi radialis' (channel 3 and 4)

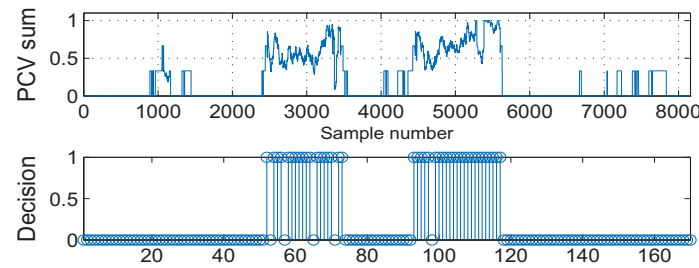

(b) BEMD phase synchrony - 'flexor carpi radialis' (channel 3 and 4)

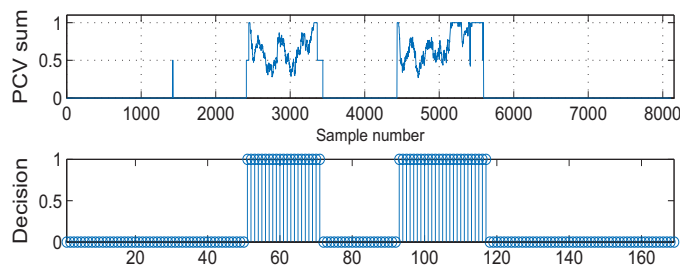

(c) MEMD phase synchrony - 'flexor carpi radialis' (channel 3 and 4)

Fig. 2. EMG data, phase synchrony, and the decision for muscle - 'flexor carpi radialis (FCR)' between BEMD and MEMD method.

TABLE I

State Table for Action Decisions using BEMD and MEMD

\begin{tabular}{|c|c|c|c|c|c|}
\hline $\begin{array}{c}\text { Syn. Pair } \\
\text { (Muscles) }\end{array}$ & $\begin{array}{c}\text { S1 } \\
\text { Ext. }\end{array}$ & $\begin{array}{c}\text { S2 } \\
\text { Flex. }\end{array}$ & $\begin{array}{c}\text { S3 } \\
\text { Pron. }\end{array}$ & $\begin{array}{c}\text { S4 } \\
\text { Sup. }\end{array}$ & $\begin{array}{c}\text { S5 } \\
\text { Null }\end{array}$ \\
\hline Ch1,2 (ED) & 1 & 0 & 0 & 0 & N/A \\
Ch3,4 (FCR) & 0 & 1 & 1 & 0 & N/A \\
Ch5,6 (PT) & 1 & 0 & 1 & 0 & N/A \\
Ch7,8 (BB) & 0 & 1 & 0 & 1 & N/A \\
\hline Act.Ind & $\mathbf{1}$ & $\mathbf{2}$ & $\mathbf{3}$ & $\mathbf{4}$ & $\mathbf{0}$ \\
\hline
\end{tabular}

muscle tensed while performing gesture of extension; FCR and $\mathrm{BB}$ muscle contracts while doing flexion; FCR and PT motivated while doing pronation; BB acted while doing supination. This theory from a medical point of view matches perfectly with the state table shown in Table I, thus providing a sound foundation of the phase synchrony approach using MEMD method.

\section{CONCLUSIONS}

We have proposed an EMD-based phase synchrony analysis for the detection of muscle contraction as a potential tool for robotics. By observing cross-information and the phase synchrony of muscle activities within an adaptive framework using multivariate extension of EMD (MEMD), it is possible to accurately identify different gestures of the arm. The 

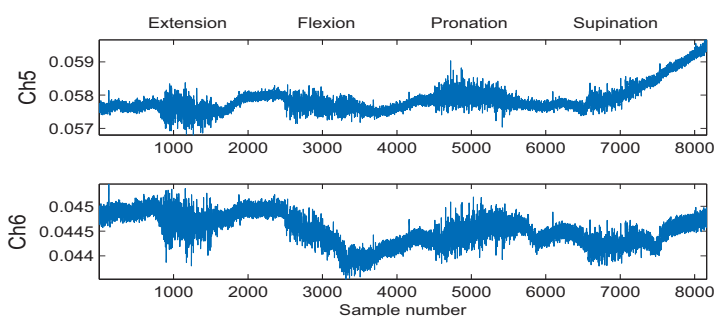

(a) Input signal - 'pronator teres' (channel 5 and 6)
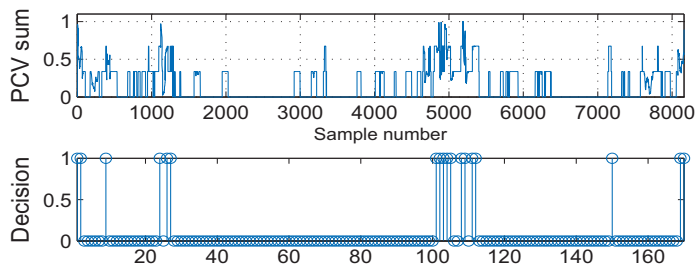

(b) BEMD phase synchrony - 'pronator teres' (channel 5 and 6)
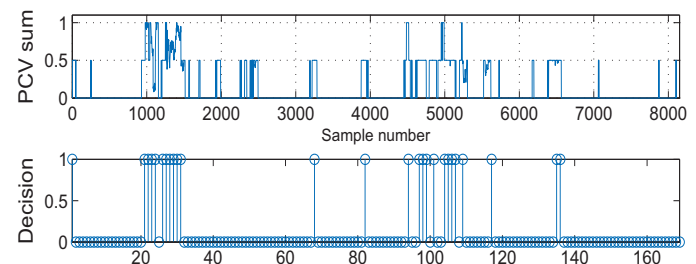

(c) MEMD phase synchrony - 'pronator teres' (channel 5 and 6)

Fig. 3. EMG data, phase synchrony, and the decision for muscle - 'pronator teres (PR)' between BEMD and MEMD method.

proposed approach is power independent and more robust to artifacts and muscle fatigue than existing methods, making it ideal for the muscle activity detection and control of mobile devices.

\section{REFERENCES}

[1] P. K. Artemiadis and K. J. Kyriakopoulos, "An EMG-based robot control scheme robust to time-varying EMG signal features," IEEE Transactions on Information Technology in Biomedicine, vol. 14, no. 3, pp. 582-588, 2010.

[2] J. Bhattacharya and H. Petsche, "Phase synchrony analysis of eeg during music perception reveals changes in functional connectivity due to musical expertise," Signal processing, vol. 85, pp. 2161-2177, 2005.

[3] N. E. Huang, Z. Shen, S. R. Long, M. L. Wu, H. H. Shih, Z. Quanan, N. C. Yen, C. C. Tung, and H. H. Liu, "The empirical mode decomposition and the Hilbert spectrum for nonlinear and non-stationary time series analysis," Proceedings of the Royal Society A, vol. 454, pp. 903-995, 1998.

[4] C. M. Sweeny-Reed and S. J. Nasuto, "A novel approach to the detection of synchronisation in EEG based on empirical mode decomposition," Journal of Computational Neuroscience, vol. 23, pp. 79-111, 2007.

[5] D. Looney, C. Park, P. Kidmose, M. Ungstrup, and D. P. Mandic, "Measuring phase synchrony using complex extensions of EMD," in Proceedings IEEE 15th Workshop on Statistical Signal Processing (SSP2009), 2009, pp. 49-52.

[6] M. Altaf, T. Gautama, T. Tanaka, and D. P. Mandic, "Rotation invariant complex empirical mode decomposition," in Proceedings of the International Conference on Acoustics, Speech and Signal Processing (ICASSP), vol. 3, 2007, pp. 1009-1012.
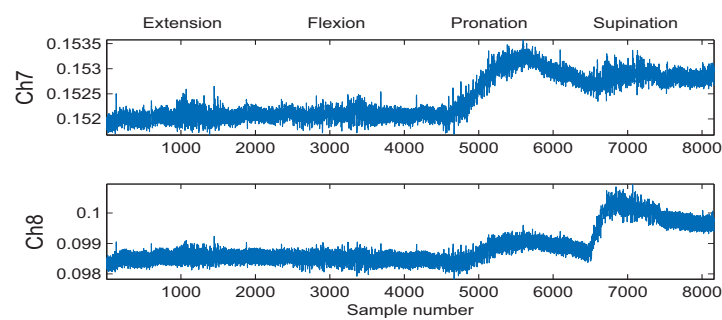

(a) Input signal - 'biceps brachii' (channel 7 and 8)
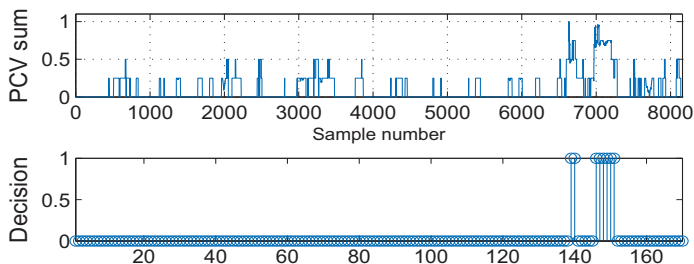

(b) BEMD phase synchrony - 'biceps brachii' (channel 7 and 8)
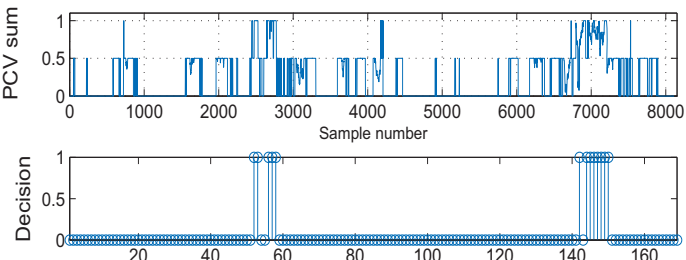

(c) MEMD phase synchrony - 'biceps brachii' (channel 7 and 8)

Fig. 4. EMG data, phase synchrony, and the decision for muscle - 'biceps brachii (BB)' between BEMD and MEMD method.
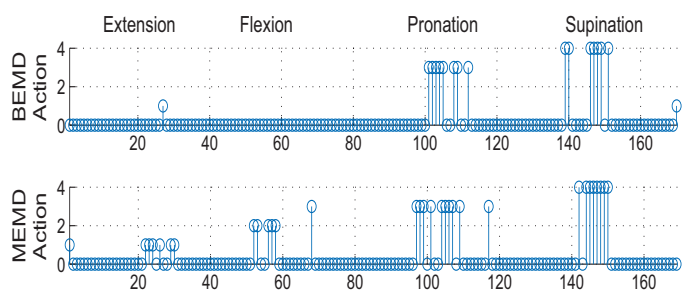

Fig. 5. Action index for the controlling of the robot.

[7] G. Rilling, P. Flandrin, P. Gonalves, and J. Lilly, "Bivariate empirical mode decomposition," IEEE Signal Processing Letters, vol. 24, pp. 936-939, 2007.

[8] N. Rehman and D. P. Mandic, "Multivariate empirical mode decomposition," Proceedings of the Royal Society A, vol. 466, pp. 1291-1302, 2010.

[9] — "Empirical mode decomposition for trivariate signals," IEEE Transactions in Signal Processing, vol. 58, pp. 1059-1068, 2010.

[10] S. Hahn, Hilbert Transforms in Signal Processing. Artech House, 1996.

[11] P. Tass, M. G. Rosenblum, J. Weule, J. Kurths, A. Pikovsky, J. Volkmann, A. Schnitzler, and H.-J. Freund, "Detection of $n: m$ phase locking from noisy data: Application to magnetoencephalography," Physical Review Letters., vol. 81, no. 15, pp. 3291-3294, Oct 1998.

[12] G. Rilling, P. Flandrin, and P. Gonalves, "On empirical mode decomposition and its algorithms," in IEEE-EURASIP Workshop Nonlinear Signal Image Processing (NSIP), 2003.

[13] R. L. Drake, W. Vogl, and A. W. M. Mitchell, Gray's Anatomy for Students. Elsevier, 2005. 